\title{
Metamorphosen - (un-)gewisse Gedanken zur Geschlechtsidentität
}

\author{
Harald Kamm
}

Angenommen: 14. Januar 2022 / Online publiziert: 28. Februar 2022

(C) The Author(s), under exclusive licence to Springer Medizin Verlag GmbH, ein Teil von Springer Nature 2022

Zusammenfassung Diese Arbeit untersucht das irritierende Bewusstwerden des kulturell bedingten Verhaftetseins der eigenen Wahrnehmungen von Geschlechtsdifferenz und -identität. Thematisiert werden die Verunsicherungen, persönlicher und auch theoretischer Art, wenn wir das Geschlechtliche nicht nur als biologisch naturgegeben wie noch bei Freud, sondern ebenso als sozial, kulturell und historisch konstruiert betrachten. Diese Irritationen und (Un-)Gewissheiten im Geschlechtlichen, die sich als Gewissheiten tarnen, zeigen sich in der psychoanalytischen Behandlung eines Transmannes und aktualisieren sich in der Gegenübertragung des Analytikers. Gender-Irritationen und (Un-)Gewissheiten in sich halten zu können, wird als eine ebenso wichtige Aufgabe gesehen wie das Vermeiden eines Abrutschens in Pathologisierung und Normierung des Geschlechtlichen im Denken des behandelnden Psychoanalytikers und im psychoanalytischen Diskurs.

\footnotetext{
Überarbeiteter Text eines Vortrags auf der Jahrestagung der DPG ,,(Un-)Gewissheiten Psychoanalyse zwischen Common Ground und Diversität“, 14. bis 16. Mai 2021, Onlinetagung aus Göttingen.
}

Der Beitrag behandelt Erfahrungen, die für Mitglieder der LGBTIQ+-Community problematisch sein können. Er soll keine Personen auf Grundlage des Aussehens, der sexuellen Orientierung oder der Geschlechtsidentität diskriminieren. Der Autor steht für die Vielfalt und ist offen gegenüber allen Personen.

Dr. rer. biol. hum., Dipl.-Psych. Harald Kamm ( $₫)$

Bamberg, Deutschland

E-Mail: harald.kamm@bnv-bamberg.de 


\title{
Metamorphoses-(un)certain thoughts on gender identity
}

\begin{abstract}
This work examines the irritating awareness of the culturally conditioned attachment of one's own perceptions of gender difference and identity. The article addresses the uncertainties both personal and also theoretical, when we consider gender not only as biologically natural as in Freud but also as socially, culturally and historically constructed. These irritations and (un)certainties in gender, which masquerade as certainties, become apparent in a psychoanalytic treatment of a trans man and become active in the analyst's countertransference. Being able to hold gender irritations and (un)certainties within oneself is seen as an equally important task as avoiding a slide into pathologization and standardization of the gender in the thinking of the treating psychoanalyst and in psychoanalytic discourse.
\end{abstract}

\section{Adam und Eva}

Es ist nun einige Jahre her, dass ich im Anschluss an eine Tagung von einem befreundeten russischen Professor für Kunstgeschichte gebeten wurde, ihm den Modeneser Dom, die berühmte romanische Kathedrale, zu zeigen, die er noch nie gesehen hatte. So standen wir also vor der Fassade der Kathedrale mit ihren meisterhaften Basreliefs des Bildhauers Wiligelmus. Erstaunt blickte mich der Freund an: „Was ist das?“ Ebenso erstaunt, um nicht zu sagen fassungslos, blickte ich zurück: „Kennst Du das nicht? Das sind Szenen aus der Genesis, Adam und Eva, die Vertreibung aus dem Paradies, die Erbsünde, Noah." Ich war in meinem Inneren, den Moment der Irritation und Verstörung übergehend, rasch zu dem Schluss gekommen, dass ich es mit einem Kunsthistoriker zu tun haben müsse, dem sich diese uns so vertraute Ikonographie nicht erschloss. Vermutlich hatten, weil er in Zeiten der Sowjetunion sozialisiert worden war, biblische Inhalte in seiner Bildung keine Rolle gespielt, so jedenfalls meine damalige voreilige Hypothese.

In der Rückschau sehe ich die Situation etwas anders und bedauere es, die Irritation unter Rückgriff auf mir vertraute kulturelle Schemata rasch kategorisiert zu haben, anstatt mich auf den Moment der Verstörung einzulassen und dem Geschehen nachzuspüren. Wie gern hätte ich ihn aus heutiger Perspektive gefragt: ,Was siehst Du eigentlich?" Vielleicht hätte er mir geantwortet, dass er diese Ikonographie nicht kenne, weil in seiner Bildungsgeschichte die Bibel keine Rolle gespielt habe. Aber vielleicht hätte er mir auch etwas ganz anderes erwidert, dass er irritiert oder gar verstört sei, an der Fassade einer Kathedrale nackte Menschen auszumachen, dass sich ihm der Sinn des Dargestellten nicht erschließe, dass er zwei Menschen sehe, die ihre Blöße verhüllen, die nicht eindeutig als Mann oder Frau erkennbar seien.

Ganz wie in „Adam und Eva“ von Samuel Butler (2005), dem viktorianischen Gelehrten und Nonkonformisten. Dort heißt es: „Ein kleiner Junge und ein kleines Mädchen betrachteten ein Bild von Adam und Eva. ,Wer davon ist Adam, und wer Eva?' fragte das eine Kind. ,Ich weiß nicht, 'sagte das andere. ,Aber ich wüßte es, wenn sie angezogen wären“" (Butler 2005, S. 74f). Man könnte diese Episode auch als Möglichkeit auffassen, die konstruktivistischen Aspekte der Geschlechtsidentität auszuloten; eine Möglichkeit, die ich allerdings verpasst habe. 
„Männlich oder weiblich ist die erste Unterscheidung, die Sie machen, wenn Sie mit einem anderen menschlichen Wesen zusammentreffen, und Sie sind gewöhnt, diese Unterscheidung mit unbedenklicher Sicherheit zu machen“, so Freud (1933a, S. 120f) in einer seiner mit „Die Weiblichkeit“ überschriebenen Vorlesungen zur Einführung in die Psychoanalyse. Das ,Tatsächliche existiert für den Menschen erst, sofern es ,gesehen' wird, sofern es gewußt, durch ein Wort bezeichnet und damit gedacht wird“" (Snell 1975, S. 18).

Doch spätestens Laqueur „,brachte mit aller Deutlichkeit die wissenschafts- und medizingeschichtliche Tatsache zum Bewußtsein, daß eine an den im heutigen Sinne ,biologischen' Körper ursächlich gebundene Zweigeschlechtigkeit ein Konstrukt des 18. Jahrhunderts ist, das in der psychoanalytischen Beschreibung der Sexualität seine moderne Fortsetzung fand. Erst diese Wahrnehmung der historischen Bedingtheit unseres eigenen Modells ermöglicht einen geschärften Blick auf die Konstruktion von Geschlechterdifferenz, Sexualität und Körperlichkeit in anderen Kulturen und weiter zurückliegenden Epochen“ (Waldner 2000, S. 1).

Es gibt mittlerweile mehrere Sammelbände zur Geschlechtsidentität (Belkin und White 2020; Hutfless und Zach 2017; Lemma und Lynch 2019) und ausgezeichnete Übersichtsarbeiten, beispielsweise von Capozzi (2021a, b), Ermann (2021) und Schweizer (2021). Ebenso gibt es mittlerweile psychoanalytische Behandlungsberichte, beispielsweise Warneke (2020). Die Bandbreite der Diskussion reicht von der Auffassung, dass das psychoanalytische Denkgebäude gefährdet sei, weil Transidentität die strukturierende Rolle des Ödipuskomplexes gefährde (Limentani 1979) bis hin zu einem entschiedenen Eintreten für eine Entpathologisierung, sodass der spezifische Umgang mit dem Thema Geschlechtsidentität nicht länger analysiert werden sollte (von Klitzing 2018; Rauchfleisch 2017, 2019). Generell geht es um ,um den Wandel von einer normativen Sozialisierung des Sexuellen zu einer selbstbestimmten höchstpersönlichen Entfaltung vielfältiger Erscheinungsformen, das heißt von der Sexualität hin zu Sexualitäten“ (Ermann 2021).

\section{Andrea}

Andrea, ein Mitdreißiger, ist ein athletischer Mann, er trägt einen Vollbart, spricht mit tiefer Bassstimme. Muskelbepackt bewegt er sich durch den Raum. Von Beginn an spielt seine physische Präsenz eine große Rolle in unseren Begegnungen. Mangels Perspektiven kam er als Krankenpfleger aus Italien, um sich in Deutschland eine Existenz aufzubauen, doch es fällt ihm schwer, Anschluss zu finden. Er fühlt sich isoliert, es gelingt ihm nicht so recht, Kontakte zu knüpfen, und er wird zunehmend depressiv. Insbesondere gelingt es ihm nicht, mit den deutschen Frauen, die so ganz anders seien, klarzukommen, Freundschaften zu schließen, eine Freundin zu finden. Sicherlich liege das an den kulturellen Differenzen, er wisse sich keinen Rat mehr und werde immer depressiver. In der Tat erzählt mir Andrea von Verliebtheiten, Tändeleien, die allerdings nie beantwortet worden seien. Die Frauen, die er schildert, erscheinen mir verfolgend, entwertend und intrusiv.

Kommt es zu intimeren Begegnungen, beispielsweise einem romantischen Abendessen, scheitern diese aus mir nicht wirklich nachvollziehbaren Gründen: 
Ich verstehe zwar die Worte in Andreas' Schilderungen, aber es ist, als könnte ich mir keinen tieferen Bedeutungsgehalt, keinen wie auch immer gearteten psychodynamischen Sinn erschließen, sodass diese Episoden für mich letztlich merkwürdig rätselhaft sind und dies auch bleiben. Beispielsweise fühlt sich Andrea ganz besonders zu einer Arbeitskollegin hingezogen. Man verabredet sich, beide werfen sich in Schale, gehen gemeinsam aus, kommen sich näher. Just, als ich - beider Scheu in Rechnung stellend - denke, dass es doch zumindest zu einem ersten Kuss kommen könnte, eröffnet mir Andrea, dass der Kontakt aus ihm, und auch mir, nicht wirklich nachvollziehbaren Gründen abgebrochen worden sei.

Unsere Gespräche muten seltsam oberflächlich, stereotyp, klischeehaft an, als gäbe es nur rudimentäre Vorstellungen von Beziehung, wie angelesen oder einer Fernsehserie entnommen, auf imitatorischer Basis, die aber nicht wirklich lebendig gestaltet werden, sondern eher holzschnittartige kulturelle Schemata zu bleiben scheinen und jeglicher Tiefe und Authentizität ermangeln.

Die Stunden erscheinen mir lang gezogen und zunehmend langweilig, nichts scheint sich zu bewegen. Häufiger führen wir Gespräche, die ich irgendwann als „typische Männergespräche“ zu beschreiben beginne. Wir unterhalten uns über Motorräder, Muckibuden; eine wichtige Rolle spielen Fitness, Trainingsmethoden etc.

Diese ,typischen Männergespräche“ geraten immer eintöniger. Zunehmend macht sich in mir Unmut breit, der sich zunächst auf der körperlichen Ebene äußert. Die Bewegungen Andreas' erscheinen mir zunehmend ungeschlacht - waren sie das nicht schon immer? - und ich entdecke/ertappe mich dabei, wie ich in mir entwertende Gedanken formuliere: „Mein Gott, wie er sich bewegt, das sind doch viel $\mathrm{zu}$ viele Muskeln, die jegliche elegante, geschmeidige Bewegung eher verhindern als erleichtern." Ich identifiziere mich auf einer körperlichen Ebene mit Andrea, versuche, seinen Körper und seine Bewegungen in mir nachzuempfinden, etwas, was mir aus meiner sportlichen Aktivität sehr vertraut ist. Immer mehr stellt sich eine körperliche Aversion ein, ich kann und will mich nicht mehr identifizieren, der Körper Andreas' und seine Bewegungen werden mir zunehmend fremd, in mir immer weniger nachvollziehbar, seine Motorik wirkt immer irritierender, um nicht zu sagen verstörend. Überhaupt ist alles ,viel zu rund“. Dieses ,viel zu rund“, das ich überhaupt nicht verstehe und in mir gebetsmühlenhaft wiederhole, begleitet mich lange Zeit. Innerlich bin ich auf große Distanz gegangen, bis ich schließlich Andrea auf körperlicher Ebene als ausgesprochen unangenehm erlebe und sehr gefordert bin, dieses Gefühl zunehmender Ablehnung in mir zu halten. Überhaupt erscheint mir meine Containment-Funktion in hohem Maße gefordert.

Etwa eineinhalb Jahre analytischer Arbeit sind verstrichen, und der repetitive Charakter der Stunden wirkt in seiner Eintönigkeit narkotisierend. Die Biografie spielt kaum eine Rolle; evident sind eine vernachlässigende Umgebung, unzulängliche Primärobjekte; die jeweils geschilderten aktuellen und vergangenen Ereignisse muten geschichtslos und im Grunde genommen eindimensional an. Selbstverständlich stelle ich Überlegungen zur Übertragung an, aber ich mache das gewissermaßen aus einem Pflichtgefühl heraus. Nicht aus einer lebendigen Anteilnahme und Interesse an Andrea gespeist, fühle mich zu einem bloßen Gefäß reduziert, das die Geschichten Andreas aufnimmt, hält, aber nicht transformiert. Innerlich gelingt es mir nicht, das Material auch hinsichtlich seiner Beziehungsdimension zu erfassen, geschweige 
denn zu verstehen. Ich muss allerdings auch einräumen, dass die ,typischen Männergespräche“ in ihrer Stereotypie einen gewissen Reiz auf mich ausüben, etwas, das ich in dieser Form gar nicht kenne, der ich wohl immer allzu beflissen war, zu differenzieren, kulturelle Schemata zu verfeinern, um nicht im Groben, Oberflächlichen eines Stammtisches hängen zu bleiben.

Hilfe finde ich bei der Konzeptualisierung des Interpsychischen von Bolognini (2014), scheint es sich mir doch nicht um eine intersubjektive Arbeit zu handeln, da das Subjekt erst noch entstehen muss, sondern vielmehr um eine interpsychische Arbeit.: „Ich fühle mich allenfalls implizit berufen, vor allem wie ein präpersonaler Zeuge/Container zu funktionieren, und mein Beitrag scheint hauptsächlich darin zu bestehen, dazubleiben, zu existieren“ (Bolognini 2014, S. 65). Aber ich bin anwesend, und Andrea legt ersichtlich großen Wert auf unsere Sitzungen, ohne dass ich das mit dem Gefühl verbände, dies wirklich zu verdienen; zu karg und oberflächlich sind meine Interventionen.

Immer mehr frage ich mich, wohin das führen soll, als es in einer Sitzung urplötzlich aus Andrea herausbricht. Er wolle mir schon lange etwas sagen, was er aber bislang nicht zu äußern gewagt habe. Etliche Male sei ich auf die Probe gestellt worden, aber erst jetzt wage er es, das, was ihn wirklich beschäftige und oft genug quäle, zu benennen. Nur kurz komme ich dazu, mich zu fragen, was es wohl sein mag, was Andrea mir gestehen/erzählen möchte. In rasender Geschwindigkeit formuliert er Sätze, die förmlich über mich hinwegrollen: Er sei früher eine Frau gewesen und habe sich in seinen jungen Erwachsenenjahren entschieden, sich einer Hormonbehandlung zu unterziehen und operieren zu lassen, um auch körperlich ein Mann zu werden. Schon als kleines Mädchen habe er sich als Junge gefühlt, fremd im Körperkäfig eines Mädchens gefangen. Die Pubertät sei der Horror gewesen, erleben zu müssen, zu einer Frau, die er nicht sei, werden zu müssen.

In einer Stunde erfahre ich mehr von Andrea, als in den eineinhalb verstrichenen Jahren, als habe sich alles in ihm aufgestaut und könne sich erst jetzt einen Weg bahnen, nachdem hinreichend geprüft worden war, dass ich hinreichend vertrauenswürdig sei. Innerhalb weniger Minuten erfahre ich alles über Hormonbehandlung, Entfernung der Brüste, der Gebärmutter, den Penisaufbau, fühle mich überschwemmt und völlig verstört. Wie kann es sein, dass ich nie etwas wahrgenommen habe? Welche kollusiven Momente haben eine entscheidende Rolle gespielt? Welche komplexen Reaktionen und affektiven Interaktionen bilden sich zwischen den Teilnehmern der analytischen Begegnung ab? Wie Schuppen fällt es mir von den Augen, die etwas kleinen Hände, die etwas breiten Hüften, die Form der Beine, die etwas schmalen Schultern; eine Erklärung für all das Runde, das ich innerlich so abwerten und von mir fernhalten musste.

Förmlich hinweggefegt, kaum des Denkens fähig, steigen Bilder von gemarterten, malträtierten, verstümmelten Frauen in mir auf. Insbesondere begleitete mich die Legende der Heiligen Agatha von Catania (225-250) über viele Sitzungen: „Die Legende schildert Agatha als eine wohlhabende, adlige sizilianische Jungfrau von großer Schönheit. Sie wies die Brautwerbung des Statthalters Quintianus zurück, da sie Christin sei; dieser nutzte den kaiserlichen Erlass zur Christenverfolgung, ließ sie verhaften und ins Bordell der Aphrodisa bringen, damit sie zur Unzucht verführt werden sollte. Agatha jedoch versuchte das erst gar nicht; der Statthalter veranlasste 
Verhöre, Folter und sadistische Qualen: Mit den Händen an einen Balken gehängt, wurden Agatha die Brüste mit einer Zange zerrissen, mit einer Fackel gebrannt und schließlich abgeschnitten. In der Nacht erschien ihr dann Petrus im Kerker mit heilendem Balsam, aber sie wies die Erquickung zurück. Tags darauf legte man sie auf spitze Scherben und glühende Kohlen, bis ein Erdbeben die Stadt Catania erschütterte, worauf das Volk Quintianus bedrohte, bis der von Agatha abließ und sie ins Gefängnis warf, wo sie starb“ (Kirschbaum 1968-1976; Glötzner 2011).

Ich betrachte Andrea, stelle ihn mir unwillkürlich als Frau vor, mit langen blonden Haaren, die sein Gesicht umrahmen, sein Antlitz umschmeicheln, mit Brüsten, einen Rock tragend. Hätte ich ihn/sie attraktiv gefunden, ihn/sie begehren können? Ich weiß es nicht, alles verschwimmt und wird unklar. Und ich frage mich, ob ich wohl immer in diesem Ausmaß an den Geschlechtsorganen meines Gegenübers interessiert bin wie in der Begegnung mit Andrea. Lemma (2014) betont als ein dynamisches Phänomen des transgeschlechtlichen Erlebens ,das ,Gesehenwerden“, vom anderen in einem inkongruenten Zustand visuell und mental wahrgenommen zu werden (oder auch nicht)" (Lemma 2014, S. 68), ohne damit eine umfassende Erklärung der Transidentität zu beabsichtigen.

Unsere Gespräche drehen sich lange Zeit um erfahrene Kränkungen und Pathologisierungen, wie fremd er sich in seinem Körper gefühlt habe, wie oft ihm die zahllosen Gutachter Fragen gestellt hatten, ob er lieber mit Puppen oder Autos spiele, gerne koche etc. Ich denke daran, dass ich leidenschaftlich koche, muss ich mir jetzt Sorgen um meine Geschlechtsidentität machen? Und jenes Puppenspiel in fernen Kindheitstagen mit Iris, dem hübschen Mädchen aus dem Nachbarhaus, dem ich nahe zu sein wünschte? Wie banal die erfahrenen/erlittenen gutachterlichen Versuche anmuten, eine eindeutige Zuordenbarkeit innerhalb eines heteronormativen Modells vorzunehmen.

Winnicott (1994) erkannte die Herausforderung, die die Verankerung der Psyche im Körper mit sich bringt, als er schrieb: „Wir gehen mit größter Selbstverständlichkeit davon aus, daß die Psyche ihren Platz im Körper hat, und vergessen dabei, daß auch dieses Wohnen der Psyche im Körper einen Entwicklungsschritt darstellt, eine Errungenschaft, die keineswegs allen Menschen zuteilwird“" (Winnicott 1994, S. 178)

Die sich anschließende analytische Arbeit bestand hauptsächlich darin, (auto)pathologisierende Schichten abzutragen, um das körperliche Erleben Andreas' zunehmend nachvollziehbar werden zu lassen. Damit wurde ein nichtstigmatisierender Raum für sein Leid, das in der Diskrepanz zwischen Körper und psychischem Empfinden liegt, eröffnet und das klinische Phänomen aus dem „Register von Gut und Böse, Normal und Abnormal“" (Klitzing 2018, S. 214) herausgelöst. Von großer Bedeutung ist es, sich im Austausch mit einem lebendigen Gegenüber, dem Analytiker, wiederzufinden, dem es hinreichend gelungen ist, nicht nur einen ,richtigen“ Körper zu haben, sondern auch Körper bzw. Leib zu sein. „Ein Mensch ist immer zugleich Leib [...] und hat diesen Leib als diesen Körper" (Plessner 1970, S. 43). Dann wird auch das Thema der Verstörung Raum finden, die Verstörung, sich im „falschen“ Körper zu befinden, aber auch Verstörung im anderen zu induzieren, um die man sehr wohl weiß und mit der man rechnet, aber eben auch darauf hofft, dass sie gehalten werde und nicht in Ablehnung münde. Und auch der Neid auf denjenigen, 
der über den richtigen Körper verfügt, kann zunehmend durchgearbeitet werden. Andreas' Männlichkeit entwickelte sich im Laufe der Zeit weniger stereotyp, mutete weniger imitatorisch an. Schließlich verliebte er sich in eine Frau, die seine Liebe erwiderte.

Es fehlte sozusagen ein weiteres „Zuhause“: „,das Denken und Fühlen eines anderen Menschen der ... [das] im Körper lokalisierte Erleben von Ambiguität, Verwirrung und Verunsicherung [und Verstörung] hätte aufnehmen können“ (Lemma 2014). Diese Arbeit hatte der Psychoanalytiker zu leisten. „Der Patient benötigt eine Analyse, in der seine schmerzvolle Körpererfahrung gesehen und vom Analytiker metabolisiert wird. ... [D]as Bedürfnis, im gewünschten Geschlecht gesehen zu werden, [enthält] manchmal den unbewussten Wunsch, sich zu verbergen“" (D'Angelo 2021, S. 77).

\section{Teiresias}

Unterzieht man Angst und Methode in den Verhaltenswissenschaften von Devereux (1973) einer erneuten Lektüre, ist man erstaunt, wie sehr die heteronormative Welt bei ihm noch in Ordnung scheint, obwohl er gerade dem Irritierenden und Verstörenden, insbesondere im Bereich der Sexualität, so viel Raum gibt. Heute laufen wir vielleicht eher Gefahr, das Verstörende aus der Welt schaffen zu wollen, einer Art Normalisierung zu unterziehen. So reden wir einer Fluidität sensu Zygmunt Baumann das Wort, die paradoxerweise ein eindeutiges binäres Modell durch die Hintertür ausgefeilter Rationalisierungsprozesse wieder einzuführen versucht, als gäbe es kein Unheimliches, keinerlei rätselhaften Botschaften, keine Konflikte, keine Ambivalenzen, keine mühsame Identitätsbildung mehr.

„Die Vorstellung einer körpergestaltentsprechenden oder -widersprechenden Identitätsbildung erscheint demgegenüber deutlich unterkomplex und reduktionistisch“ (Quindeau 2019, S. 27). „Das Dilemma des Transsexuellen macht vielleicht auf besonders extreme Weise die Entwicklungsaufgabe deutlich, der wir uns alle stellen und für die wir alle Kompromisslösungen finden müssen, nämlich die Frage, wie der Körper, den man hat, zu dem werden kann, der man ist, oder, um mit Winnicott zu sprechen, wie er zu ,uns selbst“ werden kann. Diese zentrale Entwicklungsaufgabe wird für den Transsexuellen noch zusätzlich verkompliziert, weil er aus biologischen und/oder psychologischen Gründen das Gefühl hat, in einem Körper zu leben, den er als unerträglich verwirrend und schmerzhaft unangenehm empfindet" (Lemma 2014, S. 69f).

Im Freud'schen Modell spielt die Biologie eine entscheidende Rolle, doch schlägt Freud eine „Psychosexualität“ und keine „Biosexualität“ vor. Die Bildung der eigenen Objektbeziehungen wird davon abhängen, wie man sich mit dem Geschlechtsunterschied und der Bedeutung, die man ihm zuschreibt, auseinandersetzt. In jedem von uns dauert eine Fantasie von Bisexualität fort. Allerdings ist damit nicht der Wunsch gemeint, über die Geschlechtsattribute zweier Geschlechter zu verfügen, „sondern vielmehr die Möglichkeit, Männliches mit Weiblichem in sich zusammenzuhalten, die Aktivität mit der Passivität, die Identifizierungen mit jedem der beiden Geschlechter“ (Lingiardi und Carone 2020, S. 263). 
Wichtig erscheint mir auch eine kulturhistorische Dimension, sind wir doch alle an unseren historischen Kontext gebunden, von dem zu abstrahieren nicht ohne Weiteres möglich ist: „Kein Individuum kann sich von dem historischen Zeitraum, in dem es lebt, lossagen, genauso wenig, wie es sich jenseits von Raum und Zeit stellen kann“" (Eissler 1953, S. 107).

Laqueur (1992) hatte in seinem kulturhistorisch angelegten Werk - Auf den Leib geschrieben. Die Inszenierung der Geschlechter von der Antike bis Freud - den Versuch unternommen, die Geschichte der bildlichen und sprachlichen Darstellung des Geschlechtsunterschieds zu schreiben. Seine Grundannahme bestand darin, dass Bilder und Sprache als Hervorbringungen des sozialen Handelns die komplexen Zeichen ihrer Entstehung in sich tragen. Auf hochinteressante Weise führt uns Laqueurs Untersuchung durch Texte und bildliche Illustrationen, die von der Antike bis Freud reichen, und zeigt auf, auf welch komplexe Weise diese „Modelle“, mit kulturellen und wissenschaftlichen Anliegen verwoben sowie auf verschiedenste Weise eingesetzt und umgesetzt, in Erscheinung treten. Laqueur ,hatte ein Bezugssystem im Auge, das der Erfahrung vorausliegt und Beschreibungen generiert“ (Giuliani 1997, S. 152).

In Auseinandersetzung mit Laqueur bemängelt Maihofer (1995) das Überspringen der Erlebnisfähigkeit im Leib: „Als Ergebnis historisch bestimmter Wahrnehmungsund Redeweisen kommt der geschlechtliche Körper ausschließlich über die Wahrnehmung von außen in den Blick oder durch die Rede über ihn. Verloren geht dabei die Einsicht, daß der geschlechtliche Körper stets auch erlebt, erlitten, eben erfahren wird. Was also in Laqueurs Verständnis des „Geschlechtskörpers“ völlig unreflektiert bleibt, ist die „Geschichte der Erlebnisfähigkeit im Leib“ (Maihofer 1995, S. 33f). Hatte doch Laqueur (1992) selbst bekannt, dass ihn ,die offensichtlichste und beständigste Auslassung in diesem Buch traurig macht: eine durchgängige Darstellung von Körpererfahrung. Einige mögen sagen, daß es so gerade gut ist und daß ein Mann über den sexuellen weiblichen Körper als empfindenden und liebenden nichts wirklich Interessantes oder Authentisches zu sagen hat" (Laqueur 1992, S. 38).

Dieses Erleben, Erleiden und Erfahren am und im Körper kann auch am Mythos von Teiresias untersucht werden, wobei , die mythische Überlieferungsform als das subversiv Vielgestaltige und notorisch Unzuverlässige“ (Assmann und Assmann 1988, S. 46) gesehen werden kann.

Ovid schildert Teiresias Schicksal in den Metamorphosen (Buch III, S. 316-338). Nach der Melampodie Hesiods ,tötete ... [Teiresias] bei der Beobachtung sich paarender Schlangen einmal das Weibchen, ein andermal das Männchen und erfuhr dabei jedesmal eine Geschlechtsverwandlung ... Auf Grund dieser Erfahrung befragten ihn Zeus und Hera, ob Mann oder Frau beim Geschlechtsverkehr den größeren Genuß habe. Als T. der Frau einen 9-mal größeren Genuß zuschrieb, blendete Hera ihn, Zeus aber verlieh ihm Sehergabe und 7-fache Lebensdauer" (Ziegler et al. 1979, S. 558). Teiresias hatte etwas erblickt, das er nicht hätte erblicken dürfen.

Dazu schreibt Brisson (2002): „Sexuelle Ambivalenz war in der Antike sowohl zutiefst bedrohlich für die soziale Ordnung als auch zutiefst attraktiv. Besonders in einer Kultur, in der die Geschlechterrollen so grundlegend schienen, war Hermaphroditismus monströs, ein Vorzeichen von Unordnung. Aber in den Mythen und der Phi- 
losophie jener Welt repräsentierte er auch das Versprechen der Verschmelzung, die erhoffte Belohnung der Liebe und den Boden, aus dem die Zivilisation entsprang.“ Er ermöglicht uns in seiner strukturalistisch orientierten Analyse der Teiresias-Gestalt, diesen als Mittler/,médiateur“ innerhalb verschiedener polarer Gegensatzpaare (männlich/weiblich, menschlich/göttlich, tot/lebend) zu betrachten (Ugolini 1995). ${ }^{1}$

Verschiedentlich wird in der psychoanalytischen Literatur auf Teiresias verwiesen, doch ist die Lektüre des Mythos zumeist eindimensional, berücksichtigt nur eine Lesart und übersieht das Wesentliche des mythischen Denkens und Wahrnehmens als grundlegende symbolische Form sensu Ernst Cassirer. ,Jeder mythische Text ist ... gleichzeitig traditionelle Wiederaufnahme und individuelle Variation und damit möglicherweise immer auch schon Kritik oder zumindest Diskussion einer bereits bestehenden Erzählung. Jan und Aleida Assmann (1988) schlagen als Bezeichnung für diesen Vorgang den aus der Rhetorik entlehnten Begriff der ,Hypolepse“ vor, der die Anknüpfung an das, was der Vorredner gesagt hat, bezeichnet. Es ist offensichtlich, daß dabei mündliche Erzähltraditionen schriftlich fortgesetzt wurden, die im Übrigen die ganze Antike hindurch lebendig blieben. Für uns jedoch existieren mythische Erzählungen immer nur als - hypoleptische - Texte. Es gibt keine rekonstruierbare Urfassung - der Mythos ist tatsächlich ,ein Text, den es nicht gibt ‘. Statt dessen finden wir nur immer wieder neue ,Kristallisationen', deren Form bestimmt ist durch die sich ändernden soziokulturellen Bedingungen, denen Autor und Rezipient unterworfen sind" (Waldner 2000, S. 8). Waldner hat eine große Studie zur Geschlechterdifferenz und Initiation in Mythos und Ritual der griechischen Polis vorgelegt. Ihr Blick ist ein anthropologischer - oder auch „ethnologischer“ - der ,nach der historischen Eigenart der griechischen Mythen und Rituale fragt und dabei versucht, ,eine Annäherung über bewußte Distanzierung ' herzustellen“ (Waldner 2000, S. 1).

Was mag Teiresias bewogen haben, das im Liebesspiel umschlungene Paar mit seiner Weidenrute untersuchen zu wollen? Wissenschaftliches Interesse, Neugier? Aristoteles zufolge sind die Schlangen geschlechtlich nicht auseinanderzuhalten, wirken wie ein Körper mit zwei Köpfen. Wer dächte hier nicht an Sigmund Freud in Triest, auf der Suche nach den Gonaden des Aals. Möglicherweise versucht Teiresias, das Verstörende auszuschalten, weil er nach einer Binarität sucht und diese herzustellen versucht, indem er den Anderen, das andere Geschlecht, auch in sich, tötet, und zur Strafe in genau dieses verwandelt wird, um eine subjektive leibliche Erfahrung zu sammeln, sich den Körper auf lebendige Weise anzueignen, sodass er beider Erfahrung in sich zu tragen und zu halten vermag. Es gibt auch eine Variante, in der er auf die Frage Zeus' und Heras erwidert, dass er sich nicht erinnern könne; ein Verweis darauf, dass der fremd(-gewordene) Körper des Anderen in sich nicht gefühlt, empfunden, allenfalls in einer oberflächlichen Stereotypie nachgeahmt werden könne.

\footnotetext{
${ }^{1}$ Eine zeitgenössische Adaption des Teiresias-Mythos findet sich in dem Film Tiresia des französischen Regisseurs Betrand Bonello. Diesen Hinweis verdanke ich Gherardo Ugolini, E-Mail vom 10.05.2021.
} 


\section{Carina}

Carina, eine dreißigjährige Sozialarbeiterin, wurde von mehreren Psychotherapeuten zurückgewiesen, da sie in einer queeren Beziehung lebt. Sie sei massiv pathologisiert worden und suche verzweifelt nach einem Psychoanalytiker, nachdem die queeren Thematiken aufseiten der Therapeuten zu Irritation und Unverständnis geführt hätten. Ich antworte ihr, dass wir gerne gemeinsam über ihre Sorgen und Nöte nachdenken können.

Carina wirkt sehr lebendig; unter einem blonden Wuschelkopf blicken mich muntere, kluge Augen an. Sie beeindruckt mich durch ihren Intellekt und ihre Schärfe im Denken; gleichzeitig scheint es so, als dürfe ich nur den Kopf wahrnehmen und nicht den schlanken Körper, auf dem derselbe sitzt. Dieser Körper wirkt seltsam fragmentiert, wie aus einzelnen Gliedmaßen zusammengesetzt, die keine kohärente Einheit bilden, um flüssige, geschmeidige Bewegungen zu ermöglichen.

Mir kommen die Menschendarstellungen aus der Zeit der frühen Griechen in den Sinn, wie sie Snell (1975) beschreibt, dass es uns schwer eingehe, ,daß man einmal nicht den Körper oder Leib als solchen aufgefaßt und bezeichnet haben soll ... Daß der substantielle Körper des Menschen nicht als Einheit, sondern als Vielheit begriffen wird“" (Snell 1975, S. 17). Lemma (2014) greift diesen Aspekt auf, wenn sie schildert, dass sich Transsexuelle oft erleben, ,als bestünden sie aus ,unzusammenhängenden Teilen ", oder ,als Puzzleteile, die nicht zusammenpassen", oder sagen, sie seien ,sich selbst fremd', womit sie die beunruhigende Diskontinuität ihres Selbsterlebens unterstreichen, das sie nach ihrem ,wahren“ Körper suchen lässt - einem Körper, von dem sie erwarten, er würde sie von ihren unerträglichen Gefühlen befreien“ (Lemma 2014, S. 70).

Die Probleme Carinas potenzierten sich im Laufe der herrschenden Pandemie, der sie sich hilflos ausgeliefert fühlt. Sie wird von Ängsten gequält, sie könne jemanden anstecken oder angesteckt werden. Die Hygienevorgaben werden zwanghaft ausgestaltet und muten immer mehr wahnhaft an.

Carinas Mutter hatte nach der Geburt eine langwierige postpartale Psychose entwickelt, sodass völlig unklar ist, wer sich eigentlich um sie gekümmert hat. Zugewandte, resonante, intuitiv träumerische Caregiver treten nicht in Erscheinung; der Säugling scheitert in seinem Bemühen, das Objekt seelisch lebendig werden zu lassen; projektive Identifizierungen finden keine Resonanz.

Die Eltern sind entschiedene Anhänger der No-Vax-Bewegung, sodass Carina ohne jeglichen Impfschutz in die Welt entlassen wurde, immer in Angst, sie könne sich eine fürchterliche Krankheit einfangen, ganz entsprechend den panischen Ängsten der Mutter, der Tochter könne jederzeit etwas zustoßen. Sie solle lieber zuhause, bei der Mutter bleiben, wo man in Sicherheit sei. Die äußere Wirklichkeit ist ungemein bedrohlich, mütterliche hypochondrische Ängste überschwemmen tsunamigleich die Innenwelt Carinas, und eine maligne Objekterfahrung wird verinnerlicht. Mit Williams (2005) können wir auch von der Einverleibung eines intrusiven Objektes sprechen. Dieses wütet im Inneren Carinas, sie verletzt sich, fügt sich schwärende Wunden zu, die nicht heilen wollen.

Unter Bezugnahme auf Bion können wir von einem nahezu völligen Fehlen der Funktion eines Containers, einer Alpha-Funktion, einem Mangel an Rêverie beim 
Objekt ausgehen, sodass die emotionalen Kommunikationen Carinas auf keine transformierende Resonanz stoßen und nicht in der Tiefe aufgenommen und zurückgegeben werden, Erfahrung und Entwicklung nicht stattfinden können. Im Ausbruch der durch die coronavirus disease 2019 (COVID-19) ausgelösten Pandemie wird die bedrohliche Innenwelt deckungsgleich zur bedrohlichen Außenwelt; die BetaElemente werden potenziert.

Im Rahmen einer impliziten Behandlungspraxis (Ermann 2005), die zum Ziel hat, die Containment- und die Alpha-Funktion zu entwickeln, bemühe ich mich sehr um eine entsprechende Modulierung meiner Interventionen, in ständiger Sorge, Carina könnte in eine Psychose abgleiten. Es ist eine Gratwanderung. $\mathrm{Zu}$ viel an Deutung wird sofort bedrohlich, zu wenig lässt Carina allein inmitten ihres Gefühlstsunamis zurück. Sie verträgt keine ,starken“ Interventionen, und ich laufe Gefahr, zum Verfolger, zum Eindringling, zu einem intrusiven, retraumatisierenden Gegenüber zu werden und damit die Pathogenese zu wiederholen.

Ich bin oft verzweifelt: Wie finde ich Interventionen und dosiere dieselben, damit sie transformativen Gehalt entwickeln können, die analytische Situation zu einer „Klaviersonate zu vier Händen“ (Barale 2012) zu werden vermag? Sind meine Interventionen nicht auch eher ,interprétations-bouchons“ im Sinne von Guignard (1998)? Danach ist ,vielleicht der Drang, eine Deutung anzubringen, der uns an erster Stelle warnen sollte, besonders wenn uns die Deutung, die uns in den Sinn kommt, vollkommen klar auf der Hand zu liegen scheint. Meiner Ansicht nach ist es absolut notwendig, den von uns gespürten Drang zu intervenieren, vom Inhalt der Deutung, die uns so offenkundig erscheint, loszulösen. ... [Bisweilen] ist es in der Tat notwendig zu intervenieren, nicht aber mit einer Deutung des Inhalts, noch mit einer direkten Übertragungsdeutung, sondern über ein sorgsames Tasten danach, das fragliche Gefühl in Worte zu fassen“ (Guignard 1998, S. 45).

„Durch seine intuitiven Empfindungen nimmt der Patient auf, wie der Therapeut ,funktioniert' und produziert dies in seinen Träumen und Assoziationen (Brenman 2014; Faimberg 2009; Nissim Momigliano 1992). Das bedeutet, dass der Patient, obwohl mit Problemen belastet und verzweifelt, in der Lage ist, die Erreichbarkeit des Therapeuten und seine therapeutischen Fähigkeiten sehr spezifisch wahrzunehmen, durch das, was dieser bewusst oder unbewusst vermittelt“ (De Masi 2018b, S. 75). Es geht in diesem Falle nicht um ein „Wiederfinden der guten Objektbeziehung“ (Brenman 2014), sondern darum, im Rahmen der analytischen Beziehung (De Masi 2018b, De Masi et al. 2020) vielleicht zum ersten Mal eine hinreichend gute Objektbeziehung möglich werden zu lassen.

Die Vorstellung des emotionalen Beziehungstraumas geht mit der Betrachtung äußerer Faktoren einher. In anderen Worten: Das Trauma gehört zur Abhängigkeit. Es ist Scheitern in Bezug auf Abhängigkeit. ,Deshalb muss der Analytiker das verstörende emotionale Umfeld im Blick behalten, um für jede spezielle Geschichte ein adäquates emotionales Setting zur Verfügung zu stellen. Das klinische Problem hängt nicht nur von den direkten Auswirkungen der traumatischen Erfahrung ab, sondern vor allem auch von den indirekten, das heißt von der Gesamtheit der individuellen Antworten in Form von psychopathologischen Strukturen, die vom Trauma ausgehen"(De Masi 2018a). 
Nach einer Phase der Stabilisierung lässt Carina eines Tages zuhause die Bombe platzen: Ihr Partner sei ein Transmann. Die Familie ist völlig entsetzt und bricht endgültig mit der Tochter.

An Devereuxs zentraler These, dass Angst und Methode in einem engen $\mathrm{Zu}-$ sammenhang stünden, hat sich meines Erachtens nichts verändert, doch gilt dies auch für die psychoanalytische Theoriebildung: „Kurz, wenn wir anfangen wollen zu wissen, müssen wir mit einem Eingeständnis unseres Unwissens beginnen, das im Augenblick dieses Eingeständnisses auch schon nicht mehr unüberwindbar ist““ (Devereux 1973).

De Masi (2021) kommt zu dem Schluss, dass wir aufgefordert seien, ,,darüber zu reflektieren, nachzudenken, dass Transsexualität uns auf eine neue Art und Weise zum analytischen Nachdenken verpflichtet, ohne dass wir uns auf bereits Bekanntes verlassen können. Wenn wir uns die Entwicklung der Persönlichkeit vor Augen führen, stehen wir vor dem elementaren Rätsel des psychischen und körperlichen Selbst und dem komplizierten Durcheinander zwischen dem, was gelernt wird, und dem, was biologisch vorherbestimmt ist. Deshalb vertrete ich die Auffassung, dass solchen Untersuchungen mehr Beachtung geschenkt werden sollte, auch wenn sie sich mit Themen befassen, die im Moment normalerweise nicht zur täglichen klinischen Arbeit eines Analytikers gehören“ (De Masi 2021, S. 208).

Ein Mangel an Rêverie liegt sowohl dem Konformismus als Identifikation mit der Gruppe (sozialer Konformismus) als auch dem Konformismus in der Analyse (Festhalten an den Ideen der Meister oder der psychoanalytischen Institutionen) zugrunde. Selbst die sinnvollsten Orientierungen geraten zu „Höhlen“, in denen man aus Angst vor dem Fühlen und Denken Zuflucht sucht. Dies führt dazu, dass der Einzelne „,mit den Wölfen heult“", das heißt, sich anpasst und sich seiner eigenen Besonderheit und seinem eigenen Projekt der Individuation entzieht (Gaburri und Ambrosiano 2003).

Ich möchte hier auf die ,negative capability“ verweisen, jene negative Fähigkeit, die nach John Keats (1899) große Dichter wie Shakespeare auszeichnet und darin besteht, Zweifel, Unsicherheiten, Verwirrendes und Verstörendes in Kauf zu nehmen, auszuhalten und dem Sog zu widerstehen, diesen mentalen Zustand durch voreilige Kategorisierungen und Deutungen zu entschärfen und sich nicht nervös nach Tatsachen und Vernunft umzusehen. Angst und Methode stehen in einem engen Zusammenhang.

Damit wir nicht Gefahr laufen, unsere Analysanden mit unseren kulturellen Schemata, diesmal analytischer Provenienz, zu kolonialisieren und uns die Theorie, so aufgefeilt sich auch sein mag, zum Prokrustesbett gerät. „Die Psychoanalyse kann nur in einer radikal offenen Haltung durchgeführt werden: gleichschwebend aufmerksam die Kommunikation von unbewusst zu unbewusst zulassen (Freud 1912e, 1915e), ohne Wertung und Auswahl ein intuitives Hören und Sichöffnen ermöglichen. Das heißt: offenbleiben für die individuellen Qualitäten der psychischen Struktur und Dynamik, selbst dann, wenn sie an keinerlei Vertrautes anschließen. Vorhandene Voraussetzungen, fundierte Erfahrungen, Konzepte und Theorien so weit in den Hintergrund treten lassen, sodass jederzeit völlig Neues hervortreten kann; radikaler Verzicht auf alle teleologischen und bewertenden Momente unter 
Ausschaltung von Kategorien wie pathologisch, krank, neurotisch, pervers oder gesund, entwicklungsfördernd, normal“" (Nissen 2019, S. 83 f).

Ein merkwürdiges Paradoxon stellt sich ein. Einerseits soll die Binarität durch eine Fluidität des Geschlechts ersetzt werden, andererseits wird auf einer Eindeutigkeit der Geschlechtsidentifikation beharrt, so als genüge es, in einem männlich oder weiblich anmutenden Körper zu stecken, um sich bereits als Mann oder Frau fühlen zu können, mit einer eindeutigen, ungebrochenen Identität. So als stünde der eindeutige binäre Körper zur Verfügung, einem Avatar gleich, und als müsste man sich seinen Leib, der man ist, nicht mühsam aneignen; ein Prozess voller Konflikte, Ambivalenzen und Ambiguitäten.

Interessenkonflikt H. Kamm gibt an, dass kein Interessenkonflikt besteht.

\section{Literatur}

\section{Verwendete Literatur}

Assmann A, Assmann J (1988) Schrift, Tradition und Kultur. In: Raible W (Hrsg) Zwischen Festtag und Alltag: 10 Beiträge zum Thema „Mündlichkeit u. Schriftlichkeit“. Narr, Tübingen, S 25-49

Barale F (2012) II paziente come miglior collega: Il contributo di Luciana Nissim Momigliano alla psicoanalisi. In: Chiappano A, Ferruta A (Hrsg) Luciana Nissim Momigliano: Una vita per la psicoanalisi. Il paziente miglior collega. INSMLI - Istituto Nazionale per la Storia del Movimento di Liberazione in Italia, Roma, S 90-96

Belkin M, White C (Hrsg) (2020) Intersectionality and relational psychoanalysis: New perspectives on race, gender, and sexuality. Psychoanalysis in a new key book. Routledge, Milton Park, Abingdon, Oxon, New York

Bolognini S (2014) Inauditum / Unerhört!!! ... Gewissen, Bewusstsein, Integration: Die Analyse als postraumatische Erfahrung. In: Bozetti I, Focke I, Hahn I (Hrsg) Unerhört - Vom Hören und Verstehen. Klett-Cotta, Stuttgart, S 55-75

Brenman E (2014) Vom Wiederfinden der guten Objektbeziehung: Der Konflikt mit dem Über-Ich. In: Frank C, Ohr S (Hrsg) Vom Wiederfinden des guten Objekts. frommann-holzboog, Stuttgart, S $149-164$

Brisson L (2002) Sexual Ambivalence; Androgyny and Hermaphroditism in Graeco-Roman Antiquity. University of California press, Berkeley, Los Angels, London

Butler S (2005) Wollschwein und Tafelsilber: Notizen eines viktorianischen Querdenkers. KritBrit, Bd. 6. Stutz, Passau

Capozzi P (2021a) Di cosa parliamo quando parliamo di differenza (sessuale)?, Milano

Capozzi P (2021b) Sul genere e l'identità di genere, Milano

D’Angelo R (2021) er Mann, der ich versuche zu sein - das bin ich gar nicht. In: Münch K (Hrsg) Internationale Psychoanalyse Band 16: Trieb, Trauma und Kultur: Ausgewählte Beiträge aus dem International Journal of Psychoanalysis. Psychosozial-Verlag, Gießen, S 75-103

De Masi F (2018a) Über die Auswirkungen emotionaler Traumatisierung - Arbeiten mit schwierigen Patienten. In: Horn E, Weiß H (Hrsg) Trauma und unbewusste Phantasie. Brandes \& Apsel, Frankfurt am Main, S 79-103

De Masi F (2018b) Übertragung und die analytische Beziehung. Forum Psychoanal 34:71-80. https://doi. org/10.1007/s00451-018-0300-8

De Masi F (2021) Die Arbeit mit schwierigen Patientinnen und Patienten: Die Behandlung von schweren Neurosen, Traumata und Perversionen, von Borderline- und psychotischen Zuständen. Brandes \& Apsel, Frankfurt a.M.

De Masi F, Capozzi P, Kamm H (2020) La relazione analitica: Considerazioni sulla definizione e utilizzazione del concetto

Devereux G (1973) Angst und Methode in den Verhaltenswissenschaften. Hanser-Anthropologie. Hanser, München 
Eissler KR (1953) The effect of the structure of the ego on psychoanalytical technique. J Am Psychoanal Assoc 1:104-143

Ermann M (2005) Explizite und implizite psychoanalytische Behandlungspraxis. Forum Psychoanal 21:3-13

Ermann M (2021) Das Sexuelle und die Sexualitäten: Online-Vortrag, Psychoanalytisches Institut Berlin (PaIB) am 27.1.2021

Faimberg H (2009) „,Dem Zuhören zuhören“ und die Nachträglichkeit (1993). In: Teleskoping: Die intergenerationelle Weitergabe narzisstischer Bindungen. Brandes \& Apsel, Frankfurt a.M., S 141-158

Freud S (1912e) Ratschläge für den Arzt bei der psychoanalytischen Behandlung. GW, Bd. 8

Freud S (1915e) Das Unbewußte. GW 10

Freud S (1933a) Neue Folge der Vorlesungen zur Einführung in die Psychoanalyse. In: GW XV

Gaburri E, Ambrosiano L (2003) Ululare con i lupi: Conformismo e rêverie. (Mit den Wölfen heulen. Konformismus und Rêverie). Bollati Boringhieri, Torino

Giuliani R (1997) Körpergeschichten zwischen Modellbildung und haptischer Hexis: Thomas Laqueur und Barbara Duden. In: Stoller S, Vetter H (Hrsg) Phänomenologie und Geschlechterdifferenz. WUV, Wien, S 148-165

Glötzner J (Hrsg) (2011) Die heilige Agatha: Texte und Bilder. GRÄV, Gräfelfing

Guignard F (1998) Die Deutung ödipaler Konfigurationen in der Kinderanalyse. Psychoanalyse in Europa - Bulletin EPF, Bd. 50, S 38-46

Hutfless E, Zach B (Hrsg) (2017) Queering psychoanalysis: Psychoanalyse und Queer Theory : transdisziplinäre Verschränkungen. Zaglossus, Wien

Keats John (1899) The Complete Poetical Works and Letters of John Keats, Cambridge Edition. Mifflin and Company, Houghton, S 277

Kirschbaum E (Hrsg) (1976) Lexikon der christlichen Ikonographie. Herder, Rom, Freiburg i.B., Basel, Wien

von Klitzing K (2018) Sind psychodynamische Theorien zur Geschlechtsidentität obsolet? Kinderanalyse 26:202-216. https://doi.org/10.21706/ka-26-3-202

Laqueur TW (1992) Auf den Leib geschrieben: Die Inszenierung der Geschlechter von der Antike bis Freud. Campus, Frankfurt a.M., New York

Lemma A (2014) Der Körper, den man hat, und der Körper, der man ist: Warum es für Transsexuelle wichtig ist, gesehen zu werden. In: Mauss-Hanke A (Hrsg) Internationale Psychoanalyse, Bd. 9. Psychosozial-Verlag, Gießen, S 67-87

Lemma A, Lynch PE (Hrsg) (2019) Psychoanalyse der Sexualitäten - Sexualitäten der Psychoanalyse. Brandes \& Apsel, Frankfurt a.M.

Limentani A (1979) The significance of transsexualism in relation to some basic psychoanalytic concepts. Int Rev Psychol Anal 6:139-153. https://doi.org/10.5040/9781350967489

Lingiardi V, Carone N (2020) Die Herausforderung des Ödipus in sich verändernden Familien: Geschlechtsidentifizierungen und Zugang zur Herkunft in gleichgeschlechtlichen Elternfamilien bei Fortpflanzung mithilfe von Dritten. In: Münch K (Hrsg) Internationale Psychoanalyse, Bd. 15. Psychosozial-Verlag, Gießen, S 245-275

Maihofer A (1995) Geschlecht als Existenzweise. Zugl.: Frankfurt (Main), Univ., Habil.-Schr., 1994, Helmer

Nissen B (2019) Wenn queer quer wird - Psychoanalytisches Verstehen und Konzeptualisieren in queeren Dynamiken. In: Nissen B et al (Hrsg) Queer(es) Denken in der Psychoanalyse: Eine Kontroverse zu G. Hansbury: Das maskuline Vaginale - An der Grenze zu Transgender. frommann-holzboog, Stuttgart, S 83-98

Nissim Momigliano L (1992) Two people talking in a room: an investigation on the analytic dialogue. In: Nissim Momigliano L, Robutti A (Hrsg) Shared experience: The psychoanalytic dialogue, S 5-20

Plessner H (1970) Lachen und Weinen. In Plessner H (Hrsg) Philosophische Anthropologie (Erstveröffentlichung 1941). Fischer: Frankfurt a. M. S 11-171

Quindeau I (2019) Freuds Bisexualität im Lichte der fluiden Geschlechtsidentität. Kinderanalyse 27:21-38. https://doi.org/10.21706/ka-27-1-21

Rauchfleisch U (2017) „Trans*Menschen“, Psychoanalyse und Psychotherapie. Forum Psychoanal 33:431-445. https://doi.org/10.1007/s00451-017-0289-4

Rauchfleisch U (2019) Transsexualismus - Genderdysphorie - Geschlechtsinkongruenz - Transidentität: Der schwierige Weg der Entpathologisierung. Psychodynamik kompakt. Vandenhoeck \& Ruprecht, Göttingen

Schweizer K (2021) Weiblich, männlich, divers. Psyche 75:402-432. https://doi.org/10.21706/ps-75-5402 
Snell B (1975) Die Entdeckung des Geistes: Studien zur Entstehung des europäischen Denkens bei den Griechen. Vandenhoeck \& Ruprecht, Göttingen

Ugolini G (1995) Untersuchungen zur Figur des Sehers Teiresias. Zugl.: München, Univ., Diss., 1993/94, Narr

Waldner K (2000) Geburt und Hochzeit des Kriegers. Zugl.: Zürich, Univ., Diss., 1997, de Gruyter

Warneke S (2020) Der transsexuelle Wunsch, in sich herzustellen, was einem genommen wurde; Erfahrung in der Analyse einer transsexuellen Frau mit autistoiden Anteilen. Jb Psychoanal 81:143-166

Williams P (2005) Einverleibung eines invasiven Objekts. Psyche 59:293-315

Winnicott DW (1994) Die menschliche Natur. Klett-Cotta, Stuttgart

Ziegler K, Sontheimer W, Gärtner H (Hrsg) (1979) Der kleine Pauly: Lexikon der Antike in fünf Bänden. Deutscher Taschenbuch-Verlag, München

\section{Weiterführende Literatur}

Bolognini S (2019) Flussi vitali tra Sé e Non-Sé: L’interpsichico. R. Cortina, Milano

Guignard F (2021) The infantile in psychoanalytic practice today. International psychoanalytical association psychoanalytic ideas and applications. Routledge, London

Dr. rer. biol. hum. Dipl.-Psych. Harald Kamm niedergelassen in eigener Praxis in Bamberg, ist Lehrund Kontrollanalytiker der DPG und DGPT am Institut für Psychoanalyse Nürnberg-Regensburg. Von 2011 bis 2017 Leiter der DPG-Arbeitsgruppe Nürnberg. Lehrbeauftragter der Otto-Friedrich-Universität Bamberg und Friedrich-Alexander-Universität Erlangen, Mitherausgeber Forum der Psychoanalyse, im Herausgeberbeirat Internationale Psychoanalyse und im Editorial Board des International Forum of Psychoanalysis. Seine Interessenschwerpunkte sind: Sprache und Psychoanalyse, Geschichte der Psychoanalyse und italienische Psychoanalyse. 